\title{
Políticas públicas: Entre la deliberación y el ejercicio de autoridad
}

\author{
Quim BRUGUÉ-TORRUELLA \\ Instituto de Gobierno y Políticas Públicas / Universidad Autónoma de Barcelona \\ quim.brugue@uab.es
}

Recibido: 24/01/2014

Aceptado: 11/04/2014

\begin{abstract}
Resumen
Las políticas públicas, en el siglo XXI, se enfrentan a unos problemas crecientemente complejos e inabordables desde los parámetros tradicionales de intervención. Frente a esta constatación, el artículo explora como adaptar las políticas públicas a este nuevo contexto, dotándolas de una renovada combinación de deliberación colectiva y ejercicio de autoridad pública. El argumento, adicionalmente, se contrasta con dos casos donde se ejemplifica cómo articular una política frente a problemas tan complejos como el agua y la ubicación de las prisiones en el territorio.
\end{abstract}

Palabras clave: Políticas públicas, deliberación, gestión complejidad.

\section{Public policy: Between deliberation and the exercise of authority}

\begin{abstract}
In the twenty-first century, public policies face an increasingly complex and intractable problems. It leads to the so called policy failure. Taking that situation as a starting point, the article explores how public policies can adapt to this new context, giving them a renewed combination of collective deliberation and exercise of public authority. The argument, in addition, is tested with two cases that exemplify how to articulate a policy against complex problems as water and the location of prisons in the territory.
\end{abstract}

Key words: Public policy, deliberation, managing complexity.

\section{Referencia normalizada}

Brugué-Torruella, Q. (2014). "Políticas públicas: Entre la deliberación y el ejercicio de autoridad". Revista Cuadernos de Gobierno y Administración Pública, Vol. 1, Núm. 1: 37-55.

Sumario: Introducción. 1. El colapso de las políticas públicas. 1.1. Desbordamiento escalar. 1.2. Desbordamiento discursivo. 1.3. Desbordamiento estructural. 1.4. Desbloquear las políticas asumiendo la complejidad. 2. De la teoría a la práctica: Agua y prisiones. 2.1. El plan de gestión de las cuencas internas de Cataluña. 2.1.1. El punto de partida. 2.1.2. Asumiendo la complejidad. 2.1.3. Valoración final. 2.2. El diseño de un mapa penitenciario. 2.2.1. El punto de partida. 2.2.2. Las razones de la no decisión. 2.2.3. Asumiendo la complejidad. 2.2.4. Valoración final. 3. Deliberación y Autoridad. 4. Finalmente, la deliberación "a la sombra de la autoridad". 5. Bibliografía. 


\section{Introducción}

Las políticas públicas son las acciones de la política y, como tales, aspiran a transformar intencionalmente la realidad. Su principal vocación es modelar una realidad imperfecta, intentando que se acerque a una visión idealizada. Las políticas públicas son, en definitiva, las armas con las que la política pretende hacer efectivas sus promesas.

Pero desde hace ya demasiado tiempo, las políticas públicas están fracasando en sus intentos de construir aquellas sociedades imaginadas. La crisis de credibilidad inicia su andadura en el ámbito de la política, pero se extiende al de las políticas públicas. Nos referimos así a una doble crisis de legitimidad del sistema políticoadministrativo: aquella que afecta a los inputs del sistema y aquella que se refiere a sus outputs (Hajer y Wagennar, 2003). La primera centra la atención en los mimbres del sistema (políticos, instituciones, reglas electorales, etc.), mientras la segunda pone el acento en la irrelevancia de los impactos que generan las acciones de la política (sobre el bienestar, el desarrollo económico o el equilibrio ambiental).

En este contexto, usando los términos de Hay (2007), la política se ha convertido en "un concepto sucio" que suscita "el odio" de una ciudadanía crecientemente indignada con su malas artes y sus peores resultados. Un odio que Vallès (2011) analiza a partir de la triple percepción de la política como algo incompetente, corrupto e irrelevante. Desde la perspectiva del análisis de las políticas públicas, se han identificado las dificultades para desplegar intervenciones públicas dotadas de eficiencia y eficacia. La literatura se ha referido a los efectos paralizantes de estas dificultades acuñando el término policy failure (Bovens \& Hard, 1998).

En este artículo partiremos de esta situación de colapso de las políticas públicas y argumentaremos que, para superarla, hemos de asumir la complejidad de los problemas y enfrentarlos desde procesos decisionales que combine la lógica deliberativa con el ejercicio de autoridad pública. Una vez presentado este argumento, lo contrastaremos con dos casos prácticos: la política hídrica destinada a aprobar los planes de gestión de cuencas y la política penitenciaría que debe determinar la ubicación de las prisiones en el territorio. Los estudios de caso se refieren a la experiencia de las cuencas internas catalanas y al plan de equipamientos penitenciarios de Cataluña, aunque su lógica es trasladable a otras políticas y a otros territorios.

\section{El colapso de las políticas públicas}

Las políticas públicas están colapsando y su fracaso es el motor de la creciente indignación de la ciudadanía. El enfado que genera esta situación es ingente, pues está afectando tanto a la calidad de vida de muchas personas como a sus expectativas de seguridad y progreso. Y ante esta desconcertante situación, la ciudadanía busca canalizar la rabia acumulada hacia algún culpable identificable. Tras la crisis de 2007, los mercados quizá serían un buen candidato si no fuera porque no nos facilitan una visualización clara. Son una realidad excesivamente abstracta. La política, en cambio, nos presenta de manera cotidiana los rostros de políticos corruptos e incompetentes sobre los que descargar nuestras iras. 
Así pues, según un primer diagnóstico, los políticos son los culpables del colapso de las políticas públicas y de sus desastrosas consecuencias. Ante los frecuentes casos de corrupción y malas prácticas de los últimos años, parece inevitable aceptar esta sentencia y reconocer la necesidad de regeneración política que está emergiendo con intensidad en la agenda pública. No es este el momento de entrar en un debate sobre el futuro de la política, aunque sí de plantear una hipótesis de partida importante para entender el fracaso de las políticas públicas. Así, desde nuestra óptica, otorgar la culpabilidad del colapso de las políticas a los políticos puede ser cierto aunque es superficial. Existen causas de fondo que explican este colapso, y necesitamos identificarlas para poder articular las respuestas adecuadas.

Estas razones de fondo explicarían el colapso de las políticas públicas no sólo a través de errores coyunturales sino por una situación estructural de desbordamiento. Usamos el término desbordamiento como una forma de conectar con la metáfora de la modernidad líquida (Bauman, 2000). En el actual contexto líquido -afirmamos- tanto la política como las políticas públicas se encuentran desbordadas y hacen aguas por los cuatro costados. Intentar desplegar las intervenciones públicas que nos habían dotado de solidez durante el pasado siglo es, hoy por hoy, como intentar contener el agua con las manos desnudas, una misión imposible.

Una política desbordada genera políticas públicas impotentes. Una impotencia que tiene múltiples orígenes y diversas explicaciones, pero que puede entenderse a través de la superación de las escalas territoriales y temporales (desbordamiento escalar), del abrumador dominio de la economía sobre la política (desbordamiento discursivo) y, finalmente, de la complejidad como un rasgo estructural de nuestras sociedades (desbordamiento estructural).

\subsection{Desbordamiento escalar}

En primer lugar, la política y las políticas públicas se mueven en unas escalas que no son las dominantes; de manera que sus fracasos son como los de aquel equipo de tercera división forzado a competir en la Champions League. Usando los términos de Beck (1998), la política ha perdido su poder porque éste reside, hoy, en la capacidad de irse, de moverse de un sitio a otro sin ataduras. Aquí se encuentra el poder de las corporaciones multinacionales; en su capacidad para escaparse y evitar cualquier compromiso local. Y aquí encontramos la debilidad de la política; anclada territorialmente, inmovilizada por una tupida red de compromisos y condenada a responder localmente a aquello que se le plantea globalmente. La política no sería pues la causa sino la víctima de una situación que la condena a la impotencia, al fracaso y al descrédito.

El problema de la política es pues de escala física, aunque también sus ritmos temporales le producen importantes dolores de cabeza. La política y las políticas, por definición, se mueve en el medio o en el largo plazo. Se caracteriza por visiones de futuro que deben construirse en el tiempo, a menudo dejando que las tensiones vayan madurando y facilitando, con cierta paciencia, los períodos de cocción imprescindibles para generar algo tan ambicioso cómo son los procesos de transformación social. La política y las políticas se mueven lentamente y esta lentitud es inaceptable para la 
impaciente sociedad actual. Nadie parece dispuesto a esperar y todos nos mostramos intransigentes a la hora de exigir una respuesta inmediata a nuestros problemas. Ante estas expectativas, no queda más que esperar el fracaso de unas políticas públicas que nos mienten si prometen respuestas rápidas y nos desesperan si no lo hacen.

\subsection{Desbordamiento discursivo}

Una segunda razón de fondo de la impotencia de la política y de las políticas la encontramos en el dominio de un discurso económico que coloniza la política y la obliga a jugar en un terreno que no es el suyo. El fracaso es ahora similar al de aquel equipo de fútbol obligado a competir en un campeonato de baloncesto. La economía al menos en la versión neoclásica que se nos ha impuesto- describe un mundo donde todo se explica a partir de individuos que, de manera racional y egoísta, maximizan sus funciones de utilidad. Al trasladar este lenguaje a la política desparece, en primer lugar, la noción de interés colectivo. Bentham lo ilustraba magistralmente al afirmar que "a igualdad de placer, los bolos son tan buenos como la poesía". Y también desaparece, en segundo lugar, la posibilidad de que existan actores públicos (políticos y administrativos) preocupados por algo que no sean sus propios intereses (cargos, privilegios, remuneraciones).

Se impone un discurso inconsistente pero demoledor con la política y con sus actuaciones. Por un lado, se afirma sin demasiados matices que la política y los políticos deberían aprender de la economía y de los empresarios. Comportarse competitivamente y mostrar rendimientos. Pero, por otro lado, cuando se avanza en esta dirección se acusa a la política de olvidarse del interés público y a los políticos de comportarse de manera egoísta. Las políticas públicas viven esta contradicción con una enorme impotencia, frustrando permanentemente las expectativas de unos ciudadanos que se creen clientes o de unos clientes que se reivindican como ciudadanos (Innerarity, 2011; Ovejero, 2013). Como ciudadanos queremos prisiones, pero como clientes no las queremos cerca de nuestras casas. Como clientes queremos acceso infinito y económico al agua, mientras que como ciudadanos exigimos caudales ecológicos y el mantenimiento de los acuíferos. Un auténtico callejón sin salida.

\subsection{Desbordamiento estructural}

Finalmente, y aglutinando los puntos anteriores, la política se encuentran desbordadas por la irreductible complejidad del mundo actual. Las políticas públicas fracasan en sus intentos de moldear la sociedad por el patético contraste entre la complejidad de los problemas y la simplicidad de las respuestas (escaladas y economizadas). El modus operandi dominante de las políticas públicas ha consistido en reducir los problemas a un ámbito especializado capaz de abordarlos desde sus conocimientos sectoriales. La realidad actual, sin embargo, no parece aceptar estas simplificaciones. Es demasiado compleja, sofisticada y diversa como para someterse a las restricciones de una comprensión parcial. $\mathrm{Y}$ es aquí donde radica el principal reto para las políticas públicas.

Las instituciones públicas están estructuradas y programadas para realizar de la mejor forma posible determinadas tareas bien delimitadas, pero caen en el desconcier- 
to cuando se les reclama que diseñen programas de intervención sobre asuntos multidimensionales. En otros términos, hemos preparado a las políticas públicas para operar con eficiencia, pero nunca nadie antes le había pedido que pensarán con inteligencia sobre cómo abordar asuntos complejos (Brugué, 2009). Podemos realizar un trasvase hídrico de manera eficiente y técnicamente implacable, pero únicamente desplegaremos una política de agua inteligente cuando asumamos sus implicaciones ambientales, energéticas, económicas y sociales. Y es en esta segunda parte donde colapsan las políticas públicas.

\subsection{Desbloquear las políticas asumiendo la complejidad}

Siguiendo con nuestro hilo argumental, para desbloquear las políticas públicas será necesario abordar sus dificultades de fondo y, por lo tanto, asumir y gestionar la creciente complejidad de sus objetivos. Se trata, en otros términos, de desbordar los criterios eficientistas tradicionales y situarnos en la lógica de la inteligencia decisional. El problema práctico, en este sentido, consiste en saber cómo generar unas políticas públicas dotadas de inteligencia y no únicamente de eficiencia técnica. La respuesta no es sencilla, aunque en la literatura encontramos algunas orientaciones que nos señalan como la inteligencia únicamente florece a partir del diálogo y del intercambio (Fisher y Forrester, 1993; Healey, 1997; Pollitt, 2003). En realidad, para incorporar la complejidad en las políticas públicas hemos de poner en cuestión el paradigma ilustrado de la racionalidad tecnocrática e incorporar el debate sobre la eventual pertinencia de una racionalidad comunicativa donde el conocimiento deriva del diálogo y la interacción (Habermas, 1999).

Hemos de aclarar, sin embargo, que no usamos el término complejidad simplemente como una característica de la realidad social, sino como una perspectiva teórica emergente que define la morfología de nuestro mundo físico y social. Axeldrod y Cohen (1999) definen los sistemas complejos como aquellos donde se producen intensas relaciones entre sus componentes, mientras que Cilliers (2005) considera que la complejidad tiene que ver con la densidad de las interacciones que se producen en un sistema y con el dinamismo de las mismas. Así pues, la complejidad debe entenderse de una manera holística; es decir, sin caer en tentaciones reduccionistas o simplificadoras. La complejidad exige entender el conjunto, el cual no puede ser aprehendido a partir de una comprensión simple de las partes (Holland, 1998).

Pensando en términos de políticas públicas, asumir la complejidad significa renunciar a la estrategia habitual de recurrir al conocimiento experto. Las políticas públicas no pueden basarse en la descomposición sectorial de los problemas, sino que han de asumirlos integralmente. Al mismo tiempo, los sistemas complejos generan incertidumbre e imprevisibilidad, de manera que las políticas públicas no son verdades que resuelven objetivamente los problemas. La idea de un experto que nos proporciona la conocida y popular one best solution no tiene cabida en un entorno de complejidad.

En un entorno de complejidad, por lo tanto, las políticas públicas deben ser integrales y flexibles, han de asumir la incertidumbre y han de reaccionar permanentemente frente a situaciones de constante dinamismo. Para acercarnos a estos objetivos 
aparece la deliberación, entendida como una forma no reduccionista de generar respuestas creativas a través de la comunicación y el diálogo. Desde algunas posiciones teóricas, la deliberación sería superior a la representación o a la delegación en el momento de abordar la complejidad (Benhabib, 1996; Gutmann y Thompson, 2004; Dryzek, 2000). Usando los términos de Wagennar (2007), al aumentar la capacidad de gestionar la diversidad y las interacciones, la deliberación facilitaría el flujo de conocimiento y la coordinación necesaria para tomar decisiones en contextos de complejidad.

\section{De la teoría a la práctica: Agua y prisiones}

Con el objetivo de trasladarnos de la teoría a la práctica, en este apartado nos referiremos a dos políticas públicas específicas. Ambas políticas son complejas y, por lo tanto, exigen también de un complejo proceso decisional. Su complejidad y el nivel de confrontación que generan explican tanto la parálisis de la mayoría de los planes de gestión de las cuencas hídricas en España como los aproximadamente 15 años sin que, en Cataluña, se tomará la decisión sobre donde ubicar los nuevos centros penitenciarios.

Por otro lado, estas políticas pueden ser usadas como ejemplos de éxito a la hora de asumir y abordar la complejidad. El plan de gestión de las cuencas internas de Cataluña, a diferencia de lo que ocurría en el resto de cuencas hidrográficas española, fue aprobado cumpliendo con las previsiones establecidas por la UE. También en el ámbito penitenciario, una nueva aproximación a la toma de decisiones logró superar el bloqueo de la política y en 2005 se aprobaron las ubicaciones donde construir 5 nuevas prisiones. En los dos casos, tal como hemos apuntado en el apartado anterior, la clave para desbloquear la política fue asumir la complejidad desde un enfoque basado en la racionalidad deliberativa. Un enfoque que, a través del diálogo con los múltiples actores involucrados, no se refugiaba en las certezas técnicas sino que buscaba la interacción capaz de generar una respuesta colectivamente inteligente.

Ambos casos han sido objeto de investigaciones detalladas, aunque a continuación nos limitamos a presentar una descripción genérica y a destacar los aspectos del proceso de toma de decisiones que ahora más nos interesan; es decir, la combinación de deliberación y autoridad que -a nuestro entender- es clave para desbloquear unas políticas públicas altamente complejas. Con el fin de organizar de una forma comprensible cada experiencia, establecemos el punto de partida contextual de cada caso, presentamos de forma somera las características del proceso decisional destinado a asumir la complejidad y, finalmente, destacamos las conclusiones que consideramos más relevantes.

\subsection{El plan de gestión de las cuencas internas de Cataluña}

\subsubsection{El punto de partida}

El agua es un recurso escaso del que dependen múltiples actividades económicas, el bienestar de las personas, el equilibrio ambiental, la disponibilidad energética o la producción agropecuaria. Se requiere, por lo tanto, una política pública que asuma el 
reto de la complejidad y que busque una forma inteligente de equilibrar los diversos valores e intereses que confluyen en la gestión del agua. Nos encontramos, pues, con una política pública poliédrica y conflictiva, donde el peligro de quedar bloqueados en un entramado de intereses confrontados está muy presente.

En este contexto, la Unión Europea (UE) aprobó el año 2000 la Directiva Marco del Agua (DMA). Una Directiva que emplazaba a las múltiples autoridades hídricas de la UE a desarrollar unos planes de gestión que, siguiendo determinadas indicaciones, debían satisfacer el objetivo de mejorar la disponibilidad y la calidad de las aguas para el 2015, momento en el que se evaluaría el éxito de la política pública. Para ello se fijó el año 2009 como límite para la aprobación de los planes de medidas que se aplicarían en cada cuenca. Simultáneamente, reconociendo la complejidad de intereses y de actores que intervienen en el ámbito hídrico, la propia DMA establecía la obligación de incorporar mecanismos de participación y consulta pública en el proceso de toma de decisiones. Expresándolo en nuestros términos, la DMA asume la complejidad del problema y la necesidad de ofrecer una respuesta a través de la deliberación pública.

El gobierno de Cataluña dispone de competencias exclusivas para la gestión de su cuenca interna, de manera que, a través de la Agencia Catalana del Agua (ACA), asumió la obligación de conducir el proceso de toma de decisiones que culminó en 2010 con la aprobación en sede parlamentaria del Plan de Gestión de las Cuencas Fluviales Internas de Cataluña. Este proceso, que obtuvo un premio la ONU a la innovación democrática, se diseñó combinando el rigor técnico con la creación de amplias oportunidades de participación ciudadana y debate público. Así, tras la aprobación en 2004 de un detallado informe donde se diagnosticaba la situación de las aguas en la cuenca interna (documento IMPRESS), se inició un proceso de debate que, entre el 2005 y el 2010, incorporó a los múltiples actores vinculados a la temática hídrica.

\subsubsection{Asumiendo la complejidad}

El proceso que acompañó a la elaboración y posterior aprobación de la política de agua en Cataluña es la clave para entender cómo se desbloqueó una política asumiendo su complejidad. Comparativamente con otras cuencas hídricas españolas, nos encontramos con un caso donde la construcción deliberativa de la política permitió desbloquearla; mientras que en otras demarcaciones hidrográficas donde este proceso no se realizó el resultado fue el bloqueo, la imposibilidad de aprobar la política (Espluga, 2011). De hecho, mientras que el plan para las cuencas fluviales internas de Cataluña se aprueba en el 2010 (con algo menos de un año de retraso respecto el calendario de la DMA), la mayoría de las otras autoridades de cuenca españolas están todavía en fase de aprobación o tan solo recientemente han logrado aprobar sus planes con unos retrasos que han obligado a pagar multas a la UE y que, evidentemente, han generado los consecuentes costes de oportunidad vinculados a la no política.

El caso catalán, por lo tanto, nos permite visualizar un ejemplo donde se supera la complejidad a través de un proceso de construcción deliberativa de la política. Sin 
entrar en demasiados detalles, nos referiremos brevemente a las tres fases que estructuraron este proceso: información, debate y respuesta (Brugué, 2013).

La política hídrica no sólo afecta a múltiples intereses y se interpreta desde perspectivas diversas, sino que, además, se trata de un ámbito donde las complejidades técnicas son muy elevadas. Este es un factor muy relevante a la hora de organizar un proceso de debate público, pues se podría caer en la tentación de invitar a la ciudadanía a participar en un proceso para el cual no son competentes y sobre el cual, consecuentemente, tienen pocas cosas que decir. Con el fin de afrontar esta situación, en una primera etapa informativa, se impulsaron dos líneas de acción:

- En primer lugar, a pesar de tratarse de un proceso abierto a cualquier persona interesada en debatir sobre la política de agua, se optó por dirigirse de manera prioritaria a las diversas organizaciones vinculadas al ámbito hídrico y que, por lo tanto, disponen de conocimiento y criterio propio al respecto. Para ello se realizó un esfuerzo inicial para identificarlas (mapas de actores) y para invitarlas de manera personalizada al proceso. Tomando en consideración que en estos mapas se identificaron organizaciones muy diversas (industriales, agrícolas, ambientales, vecinales, sociales, académicas, etc.), el resultado final fue una base de datos con más de 10.000 entradas.

- En segundo lugar, el proceso de debate debía partir de materiales informativos y de una estrategia de comunicación que permitiera a los actores interesados no sólo conocer la existencia de los espacios de debate sino, también, disponer de los conocimientos necesarios para participar en él de manera efectiva. En este sentido, se trabajó en diversas direcciones: versiones simplificadas y localizadas del documento IMPRESS, materiales divulgativos diversos (DVD, trípticos, etc.), un potente sitio web donde obtener información y donde ofrecer trazabilidad del proceso, espacios de difusión en algunos medios de comunicación, etc.

Tan sólo hemos mencionado someramente estas actuaciones iniciales, pero no deberíamos olvidar que esta etapa informativa fue quizá la que generó más dedicación y absorbió la parte más significativa de los aproximadamente $3.000 .000 €$ que se dedicaron al proceso de debate público. En cualquier caso, tras esta fase informativa y comunicativa, se inició la etapa más estrictamente participativa. En ella se pretendía escuchar los argumentos de los diversos actores, propiciar su debate e intercambio y, finalmente, concretar un conjunto de propuestas para ser trasladarlas a los responsables públicos de elaborar los planes de medidas.

La organización de estos espacios de escucha y diálogo se descentralizó en 12 unidades territoriales de participación, de manera que los participantes tenían conocimientos de proximidad y, al mismo tiempo, se minimizaban las distancias y los costos de la participación. En esta dirección, se realizaron sesiones de debate en 132 de los 946 municipios existentes en Cataluña. Por otro lado, en el desarrollo del proceso de debate se distinguieron dos momentos: la validación del diagnóstico y la construcción de las propuestas. La gestión de los procesos de participación se trasladó a empresas especializadas que se responsabilizaron de su dinamización y de recoger 
las conclusiones. La administración pública, por lo que se refiere a sus directivos, estuvo presente como impulsora y receptora de las propuestas finales; mientras que el personal técnico y administrativo participó como soporte, aclarando dudas y proporcionando informaciones que los participantes consideren necesarias.

El proceso fue largo y no podemos ahora detallarlo suficientemente, de manera que nos limitaremos a ofrecer dos datos que ilustran su alcance: se realizaron 290 reuniones y participaron 1.769 personas. De estas 1.769 personas, el 29\% provenían de las administración (principalmente la local), el 30\% de entidades sociales y ambientales, el 20\% del sector económico y energético, el 13\% del ámbito agropecuario y el $8 \%$ eran participantes a título individual. Como ya habíamos mencionado anteriormente, el sitio web permitía seguir los debates y garantizaba la trazabilidad del proyecto en todos sus detalles.

Estamos relatando un proceso de toma de decisiones en una política pública y, consecuentemente, debemos preguntarnos sobre la capacidad de incidencia que ha tenido el debate público en la decisión finalmente adoptada. Plantearnos este interrogante es imprescindible para conocer la capacidad tanto de escucha como de respuesta de los responsables públicos. También sobre este aspecto nos limitamos a destacar algunos datos:

- Por un lado, las dinámicas participativas estaban diseñadas para que cada ámbito territorial concluyera con un conjunto de propuestas para, eventualmente, ser incorporadas en los planes de gestión. De este modo, la secuencia de reuniones culminaba con la presentación y el traslado de estas propuestas a un alto responsable de la ACA. De este modo, se escucharon concretamente 1.529 propuestas que, de este modo, concretaban las inquietudes y los equilibrios establecidos en los espacios de debate.

- Por otro lado, el compromiso de los responsables del ACA consistía en darse un margen de tiempo para analizar y valorar las propuestas (entre 2 y 3 meses) y convocar una nueva reunión en la que se respondía individualmente a cada una de ellas. Se garantizaba, así, que no únicamente se había escuchado sino que, además, se había valorado lo escuchado y se había respondido. Las respuestas se distribuyeron en 4 grandes categorías: las que se aceptaban pero que, en realidad, ya estaban previstas por la propia administración (40\%); las que se aceptaban y representaban algún tipo de innovación (23\%); las que se rechazaban (5\%); y las que, debido a no ser competencia de la administración convocante del proceso (la ACA), no encontraron una respuesta adecuada (32\%).

A partir de este momento, se redactó el plan de medidas, se sometió al preceptivo período de información pública y de alegaciones (que, en este caso, de manera extraordinaria, duró 6 meses) y, finalmente, como ya habíamos anticipado, el Parlamento de Cataluña lo aprobó durante el 2010, con algo menos de un año de retraso respecto las indicaciones de la DMA. 


\subsubsection{Valoración final}

Hemos intentado resumir un proceso que afecta a una política pública compleja y polémica sin introducir elementos valorativos, pero no podemos concluir sin apuntar algunos aspectos positivos y negativos de la experiencia. Lo hacemos de forma esquemática.

En cuanto a los aspectos positivos, en primer lugar, el proceso ha sido evaluado positivamente por los propios participantes, quienes destacan su rigor, la posibilidad de introducir algunos cambios en la política y, sobre todo, la oportunidad de conocerse entre los múltiples y muy diversos actores. De este modo, recuperando una idea del mundo de la teoría, se constata como los procesos deliberativos son, simultáneamente, espacios para intercambiar argumentos y para establecer relaciones de confianza mutua (Barber, 2004). Ambos aspectos tienen una incidencia relevante en la calidad tanto de la decisión como de la implementación de una política pública.

En segundo lugar, la propia aprobación del plan es un éxito, especialmente si lo comparamos con lo que ha sucedido en el resto de las cuencas españolas, donde hasta el 2012-2013 no se empezaron a aprobar los planes. El largo proceso participativo fue costoso, pero permitió a los responsables públicos tomar una decisión difícil. En otras cuencas, donde el debate público fue muy limitado, esta legitimidad no se alcanzó y, consecuentemente, fue imposible aprobar los planes. Estos retrasos comportaron multas de la UE y, evidentemente, el coste de oportunidad de no disponer de un plan de trabajo que, casi sin tiempo de aplicarse, será evaluado en el 2015.

Respecto a los aspectos negativos, como hemos anticipado en el apartado anterior, destaca aquel $32 \%$ de propuestas que no encontraron respuesta. No sólo se trata de un porcentaje importante sino de propuestas de hondo calado. La no repuesta se explica tanto por las dificultades de las relaciones interdepartamentales e interinstitucionales (que ilustran una cultura administrativa segmentada) como por la debilidad de un liderazgo político (incapaz de romper las barreras sectoriales e imponer una visión de conjunto). También fue motivo de insatisfacción la exclusión del debate de un asunto tan relevante como el precio del agua.

En definitiva, la aplicación de la DMA en las cuencas internas de Cataluña nos muestra un ejemplo práctico sobre cómo construir deliberativamente una política pública de elevada complejidad. El proceso tiene sus luces y sus sombras, de manera que se convierte, así, en una herramienta para el aprendizaje. Vinculado estas conclusiones con nuestra argumentación inicial, observamos como las luces se relacionan con la capacidad de asumir la complejidad y de abordar las decisiones públicas combinando deliberación con autoridad -debate abierto y, simultáneamente, ejercer la responsabilidad de tomar una decisión compleja y polémica. Las sombras se encuentran precisamente cuando se renuncia a tomar decisiones complicadas (como era el precio del agua) o cuando no se es capaz de incorporar la complejidad (en este caso al no poder generar un diálogo entre los diferentes departamentos del gobierno autonómico.) 


\subsection{El diseño de un mapa penitenciario}

\subsubsection{El punto de partida}

Una de las decisiones más relevantes para la política penitenciaria es aquella que atañe a la ubicación de las prisiones. Decidir dónde se construye un equipamiento penitenciario depende de múltiples variables: el tamaño previsto, la conectividad del emplazamiento, la proximidad con los familiares, las relaciones con el entorno, las reacciones de los vecinos, la seguridad, etc. Simultáneamente, la ubicación de una prisión es en sí misma una variable muy relevante para entender el modelo de política penitenciaria que se desarrolla (Wacquant, 2006). Estamos, en definitiva, como en el ejemplo anterior, presentando un caso de toma de decisiones en un contexto donde intervienen múltiples variables y donde, obviamente, se produce el cruce de múltiples valores e intereses.

Cataluña es la única Comunidad Autónoma española que, desde los años ochenta, dispone de competencias penitenciarias y, por lo tanto, dispone de capacidad política y administrativa no sólo para construir estos equipamientos sino para decidir donde construirlos. Esta capacidad legal, además, se complementa con voluntad política de ejercerla y, al menos hasta la crisis de 2007, con disponibilidad de recursos económicos para implementarla.

Junto a estas competencias y capacidades, la década de los noventa supuso una enorme presión sobre un sistema penitenciario crecientemente desbordado, donde el déficit de plazas penitenciarias causaba graves situaciones de hacinamiento. Las razones son múltiples, pero destacaríamos el incremento poblacional (alrededor de 1.000.000 más de habitantes en una zona que contaba con algo más de 6.000.000) y el endurecimiento del código penal (que nos ha convertido en uno de los países con mayor porcentaje de presos). Finalmente, también es importante recordar que la opinión pública se muestra crecientemente preocupada por lo que se considera un sistema penal excesivamente blando en lo que se refiere al cumplimiento de penas. La ciudadanía, por tanto, no se muestra reacia a la construcción de prisiones, sino más bien al contrario.

Así pues, nos encontramos en una situación donde el Gobierno de Cataluña dispone de competencias para diseñar un mapa penitenciario, de los recursos económicos necesarios para construir prisiones, de datos objetivos que avalan la necesidad de nuevas plazas penitenciarias, de voluntad política para hacerlo y de una opinión pública que, al menos en términos genéricos, se muestra bien predispuesta a ver como se construyen nuevos equipamientos penitenciarios. Y sin embargo, durante algo más de una década, la decisión de construir nuevas prisiones quedó bloqueada. Se procedió a ampliar algunos de los equipamientos ya existentes, pero no se consiguió -hasta 2006- construir nuevas prisiones en nuevos emplazamientos. 


\subsubsection{Las razones de la no decisión}

Parece extraño que, en el contexto anteriormente descrito, no se tomara la decisión de construir ninguna prisión durante más de una década. Aunque la sorpresa es relativa y, en realidad, se trata de una situación muy frecuente y que ha venido siendo analizada desde la lógica de los fenómenos NIMBY (not in my back-yard) o, usando nuestra terminología, por la influencia de la Cultura del No (Nel·lo, 2003). Nos referimos a las reacciones que se generan frente a determinadas iniciativas políticas que, por una parte, los ciudadanos reclaman $\mathrm{y}$, por otra parte, se niegan a asumir debido a las externalidades negativas que se supone pueden generar. Queremos energía eólica, per nos molestan los molinos de viento; exigimos cobertura telefónica, pero no aceptamos una antena en nuestro tejado; o, volviendo a nuestro caso, estamos a favor de encerrar a los delincuentes en prisiones, pero no permitimos que éstas se construyan cerca de nuestras casas.

No podemos ahora profundizar en una discusión sobre los efectos NIMBY y, por lo tanto, nos limitaremos a exponer, de manera muy sintética, las dos principales razones que explican el bloqueo de la decisión sobre las nuevas ubicaciones penitenciarias:

- En primer lugar, a menudo se culpabiliza a los propios ciudadanos de las dificultades asociadas a este tipo de decisiones. Los individuos, cada vez más cerrados en la defensa de sus intereses particulares, serían incapaces de entender que, a menudo, el interés colectivo exige ciertos sacrificios personales.

- En segundo lugar, los gobernantes, que ya anticipan las dificultades, tienden a tomar estas decisiones sin la imprescindible información, transparencia y diálogo con los diversos actores implicados. Se suele considerar que se trata de decisiones polémicas y que, por lo tanto, es mejor adoptarlas de manera discreta y sin generar ruido.

En el caso del mapa de equipamientos penitenciarios situamos el foco en la segunda razón. Es cierto que el creciente individualismo dificulta la adopción de determinadas decisiones (porque aparentemente podrían afectar la seguridad de mi barrio, el precio de mi vivienda o la marca de mi territorio), pero consideramos que la forma de superarlas no es culpabilizar a los ciudadanos sino modificar los procesos decisionales -asumiendo su complejidad. En realidad, hasta que no se trasladó el énfasis de la primera a la segunda explicación -a partir de 2004- la decisión continuó bloqueada. La ciudadanía únicamente permitió a los responsables públicos tomar la decisión cuando se les explicó el proyecto de manera transparente y cuando, además, se les permitió opinar y se escucharon sus miedos y sus propuestas.

Es decir, únicamente cuando se articuló una respuesta compleja (deliberativa) a un problema complejo (poliédrico) se pudo desbloquear la política. No se trataba, en definitiva, de que unos ciudadanos egoistas no permitieran la construcción de unos equipamientos públicos necesarios. Se trataba, más bien, de unos ciudadanos que no permitían que sus representantes tomaran decisiones que les afectaban desde la simplicidad, la opacidad y la incapacidad para escuchar. 


\subsubsection{Asumiendo la complejidad}

A partir de 2004, tras un cambio en la composición del gobierno autonómico, se plantea la necesidad de desbloquear el mapa penitenciario y la determinación de hacerlo a través de un proceso decisional abierto, transparente y participativo. Este proceso se desarrolla durante el año 2005 y culmina, ya en el 2006, con la aprobación de un plan que identifica 5 nuevos emplazamientos para 5 nuevas prisiones. Aunque se trata de una simplificación, podemos esquematizar el proceso decisional a partir de 5 etapas (Generalitat de Catalunya, 2006):

- Definir los criterios de la política. Una fase imprescindible -y curiosamente no realizada durante la década de la no decisión- consiste en definir los criterios políticos que orientan la ubicación de las prisiones. Sin estos criterios, las soluciones responden a criterios de oportunidad y así las perciben los ciudadanos. Es decir, no se trata de situar la prisión donde confluyen menos resistencias (porque son pocos o porque tienen dificultades económicas) sino de ubicarla en aquel territorio que cumple determinadas características. Sumariamente, en nuestro caso se consideró que las prisiones tenían que ser pequeñas, cercanas a núcleos urbanos, fácilmente accesibles y repartidas territorialmente. Detrás de estos criterios hay un posicionamiento sobre el modelo penitenciario que políticamente se pretende impulsar, una posición política que delimita los ejes del debate y que anticipa la responsabilidad pública sobre la política pública.

- El decreto de compensaciones. En este tipo de políticas suelen existir compensaciones para aquellos territorios que acogen instalaciones no deseadas, aunque la manera de determinarlas no es única. En primer lugar, las compensaciones pueden distribuirse a través de un quasi-mercado: aquel municipio que acoja una prisión tendrá determinadas contrapartidas y, a partir de aquí, que compitan. En segundo lugar, podemos reunirnos y negociar bilateralmente, de manera que las compensaciones no sean genéricas sino que se adapten a las necesidades de cada territorio previamente escogido. Y, en tercer lugar, podemos aprobar un decreto ley, de manera que cuando se tome la decisión los municipios ya sabrán las compensaciones que les corresponde. En el caso que nos ocupa, se optó por la tercera opción.

- Aprobación de los criterios por parte de la Mesa Penitenciaria de Cataluña $(M P C)$. En este tercer estadio todavía no se había decidido donde se ubicarían las prisiones, pero se disponía de criterios y, por lo tanto, se puedo someterlos a un proceso de información y debate público. Se trata de criterios políticos y genéricos que, por supuesto, no despiertan la misma polémica e interés que la decisión sobre la ubicación concreta del centro penitenciario. Esta es la razón de usar la MPC, un órgano previamente existente donde periódicamente se reúnen los diversos actores vinculados a la política penitenciaria, como espacio para debatir y posteriormente aprobar los criterios que han de satisfacerse a la hora de tomar la decisión.

- Identificación de los 5 nuevos emplazamientos. Dotados de estos criterios, en esta cuarta fase los responsables de la política penitenciaria -haciendo ejercicio de su 
posición de autoridad- decidieron los 5 emplazamientos para los nuevos centros penitenciarios. No se sometió la decisión a la aprobación de los afectados, pero cumplía con los criterios fijados en las etapas anteriores. Vemos otra vez como, para asumir la complejidad, las políticas públicas deben combinar la deliberación con la autoridad.

- El fórum "Prisiones y Territorio". Finalmente, una vez adoptada la decisión sobre el emplazamiento se convocó a los diversos actores implicados a un segundo fórum de debate. No se trataba de discutir donde se construirían las prisiones sino sobre cómo se integraba una prisión en el territorio. Para ello, las aproximadamente 100 personas que participan en el fórum empezaron identificando sus temores y prejuicios, definiendo como conjurarlos y concluyendo con una serie de reclamaciones y/o propuestas a los responsables de la política. Estos responsables, una vez valoradas las reclamaciones y propuestas, convocaron de nuevo al fórum y dieron las oportunas respuestas a las demandas recibidas. Se trataba, en definitiva, de articular una última fase donde se escuchaba a los actores y donde sus palabras eran tomadas en consideración. No todas las demandas fueran satisfechas, pero esta fase supuso algunas modificaciones que permitieron mejorar el plan de equipamientos penitenciarios.

\subsubsection{Valoración final}

Tras estas 5 fases, se aprobó el plan, desbloqueando una decisión que hacía más de una década que se posponía. No fue una decisión compartida por todos los actores, como suele pasar; pero fue una decisión dotada de suficiente legitimidad como para ser aceptada. Los ciudadanos demostraron ser capaces de entender la existencia de un interés colectivo, mientras que en el anterior enquistamiento se mostraba como no toleran que las decisiones se adopten sin explicaciones, sin transparencia y sin participación. Las decisiones complejas, en definitiva, no reclaman cierre y discreción sino abertura y diálogo.

El principal aspecto positivo de esta experiencia es la propia decisión, la capacidad para superar una situación de bloqueo que duraba ya más de una década. En este sentido, frente a algunos escepticismos, el proceso decisional deliberativo se demostró útil para abordar la complejidad y, de este modo, para que las autoridades públicas lograran la suficiente legitimidad para adoptar una decisión difícil. También desde una perspectiva micro, el proceso mostró como combinar autoridad y deliberación. El ejercicio de autoridad era imprescindible, pues es imposible lograr que todos los actores involucrados acepten satisfechos la ubicación de una prisión.

Al mismo tiempo, la deliberación no únicamente descartaba las críticas a la posible arbitrariedad de una decisión, sino que en algunos casos generó mejoras concretas en esta decisión. Por citar sólo un ejemplo, en el fórum "Prisiones y Territorio" se manifestó el temor de que uno de los emplazamientos escogidos -situado en la cima de una colina- pudiera generar una imagen de marca para el municipio. También se hicieron referencias al impacto paisajístico de una ubicación considerada ambientalmente inadecuada. Frente a estas observaciones, los responsables públicos no modifi- 
caron la elección del municipio, pero sí un cambio en el emplazamiento, trasladándolo a una pendiente que minimizaba tanto el efecto marca como el impacto ambiental.

En definitiva, nos encontramos con un ejemplo en el cual se supera el enquistamiento en una no decisión. Como en el caso anterior, la forma de hacerlo no es buscar aparentes certezas técnicas ni esconderse en los recovecos administrativos. Al contrario, el proceso decisional abre las puertas, asume la complejidad del problema y lo aborda sin falsas simplificaciones.

\section{Deliberación y Autoridad}

En la literatura más reciente sobre como gobernar sociedades complejas se nos suele plantear la necesidad de transitar desde modelos jerárquicos y autoritarios hacia modelos reticulares y participativos. Buena parte de las aportaciones sobre gobernan$z a$ argumentan en esta dirección, asumiendo la imposibilidad de ejercer las tareas gubernamentales en solitario y proponiendo formas relacionales de gobernar (Castells, 1996; Rhodes, 1997; Pierre, 2000; Fischer, 2003). También desde el ámbito de la gestión pública se ha planteado la necesidad de sustituir las visiones más tecnocráticas y gerenciales por aproximaciones que ponen el acento en el pluralismo y la colaboración (Kettl, 2002; O’Leary y Bingham, 2009; O’Leary, Van Silke y Kim, 2010).

En este artículo hemos abordado la cuestión desde el reconocimiento de la complejidad y la incorporación de la racionalidad deliberativa como única respuesta posible a esta complejidad (Mansbridge, 1983; Gutmann y Thompson, 2004; Martí, 2006). Uno de los aspectos más debatidos en la literatura sobre la racionalidad deliberativa aplicada a la definición de políticas públicas se refiere a su capacidad o incapacidad para generar consensos. Es decir, para algunos el proceso de intercambio de argumentos entre los diversos actores acabará generando un punto de acuerdo, mientras que para otros el conflicto nunca desaparece y, consecuente, se requiere completar el diálogo previo con un posterior ejercicio de autoridad.

A la luz de la experiencia empírica con políticas públicas complejas, nos situamos claramente entre aquellos que consideran que los conflictos no desaparecen y que, por lo tanto, en el diseño de políticas públicas deberemos incorporar tanto espacios deliberativos como momentos para ejercer la (legitima) autoridad pública. Y son los propios casos de estudio los que nos indican el camino para lograr este equilibrio. A nuestro entender, son tres las condiciones necesarias para avanzar en esta dirección:

1) Partir de marcos de referencias definidos políticamente:

La articulación de la deliberación con el ejercicio de autoridad reclama, en primer lugar, un marco de referencia que defina tanto el espacio del debate como los límites que la autoridad pública no está dispuesta a traspasar. En el interior del marco de referencia se debe producir la interacción deliberativa, generando las condiciones de información y respeto mutuo que la favorecen. Los debates en torno a los contenidos de los planes de gestión de cuenca o a las nuevas ubicaciones para los equipamientos penitenciarios se producen en el interior de este marco. 
Sin embargo, no debemos olvidar que este marco debe disponer de unos límites definidos políticamente. Unas fronteras que se trazan en función de disponibilidades económicas, restricciones jurídicas $\mathrm{y}$, sobre todo, de los compromisos políticos adquiridos por nuestros representantes políticos. Por ejemplo, en el caso penitenciario se opta por un modelo penitenciario que prima la reinserción y que, en consecuencia, considera imprescindible situar la prisiones cerca de entornos urbanos con los que interactuar. Podremos, por lo tanto, deliberar sobre dónde situar las prisiones si las alternativas cumplen con este requisito (están dentro del marco), mientras que no lo aceptaremos cuando se nos proponga como emplazamiento una isla desierta en medio del mar (porque queda fuera de nuestro límites). Estamos dispuestos a deliberar sobre cómo mejorar el ahorro hídrico en las explotaciones agrarias (está dentro del marco), mientras que no vamos a discutir un eventual trasvase desde el Ródano (pues, como se explicitó en el momento electoral, queda fuera de los límites).

El interior del marco representa el espació de deliberación, mientras que las fronteras nos muestran su articulación con el legítimo ejercicio de autoridad. Para poder tomar decisiones complejas necesitamos tanto ampliar los marcos de debate como delimitar claramente sus fronteras. Si el espacio deliberativo es excesivamente reducido, la deliberación pierde sentido; mientras que si las fronteras están excesivamente difuminadas, la ausencia de autoridad se traducirá, de nuevo, en parálisis de la política pública. En el arte de equilibrar el espacio con sus límites encontramos la articulación de la deliberación con la autoridad.

\section{2) Una doble obligación: escuchar y responder:}

Esta articulación también se produce en un segundo equilibrio, el que se da entre la obligación de escuchar y la obligación de responder. La primera es la base de una lógica deliberativa que pretende alimentar las decisiones públicas de aquello que expresan actores diversos, mientras que la segunda se convierte en el ejercicio de autoridad a través del cual los decisores públicos deben asumir (y presupuestar) determinadas propuestas y rechazar otras.

Los planes de gestión de cuenca se alimentaron de 290 reuniones deliberativas y generaron 1.529 propuestas, pero no todas fueran aceptadas por la autoridad hídrica. Un rechazo que no sólo se explica por quedar fuera del marco de referencia, como apuntábamos más arriba, sino sobre todo por la persistencia del conflicto. Entre las 1.529 propuestas encontramos contradicciones que expresan el inevitable y legítimo conflicto de valores o intereses. La deliberación puede equilibrar posiciones, pero no garantiza -especialmente cuando los asuntos son polémicos- que se llegue a acuerdos. En el ejemplo de las prisiones, el desbloqueo de la decisión no supuso en ningún caso que desaparecieran las plataformas anti-prisión. Decidir el emplazamiento de un equipamiento no deseado reclama debate y transparencia, pero también decisión y ejercicio de autoridad.

Mitificar la posibilidad de alcanzar consensos permite, al menos teóricamente, descartar el ejercicio de autoridad. Si hemos llegado a un acuerdo, la deliberación es suficiente y no requiere de autoridad. Sin embargo, en la medida que la política existe 
precisamente porque en la convivencia colectiva no siempre nos ponemos de acuerdo, la deliberación se convierte en un mecanismo de aprendizaje y legitimación; aunque, en la medida que no anula el conflicto, requiere de un ejercicio posterior de autoridad. En el arte de equilibrar la escucha con la respuesta encontramos una segunda clave para articular deliberación y autoridad.

3) De los clientes de servicios a los ciudadanos receptores de políticas públicas:

La tercera clave la encontramos en el paso de los clientes a los ciudadanos. Las lógicas gerenciales que se impusieron a partir de los años ochenta parecieron convencernos de que tratar a los ciudadanos como clientes era una buena idea. Pero se ha demostrado falso. En realidad se convierte en una trampa para una administración pública que se ve forzada a prometer lo imposible, a dar la razón a los que opinan blanco y a los que opinan negro y, finalmente, a fracasar en el intento patético de convertirse en una eficiente empresa de servicios.

La administración no es una empresa sino una institución pública. No presta servicios sino que ofrece políticas públicas. Y no lo hace para clientes individuales sino para el conjunto de la ciudadanía. Sin aceptar claramente estas tres premisas, las políticas públicas se muestran incapaces de superar los bloqueos que le impone la creciente complejidad del contexto en el que opera. Las política penitenciaría no puede ni maximizar la satisfacción de los vecinos-clientes ni tampoco condicionarse a las encuestas que rellenen los familiares de los presos. De la misma manera que la política de agua debe asumir la imposibilidad de satisfacer las demandas de clientes tan diversos como los ecologistas, los industriales o los agricultores.

En definitiva, la deliberación debe servir articular las voces de la colectividad, mientras que el ejercicio de autoridad debe descartar las tentaciones clientelares de determinadas visiones gerenciales de la administración pública. La piedra roseta que equilibra el peso de la deliberación y de la autoridad recae en los ciudadanos. Nunca en su clientalización.

\section{Finalmente, la deliberación "a la sombra de la autoridad"}

Al hilo de las reflexiones del apartado anterior, podemos situar el reto de articular la deliberación con la autoridad en referencia a cierta literatura sobre gobernanza. Una literatura que se han planteado las dificultades de trabajar con un aproximación de redes que excluye los mecanismos de control, dirección y, por lo tanto, sin ninguna referencia explícita a la autoridad. Deliberación entre actores sin que nadie ostente la autoridad.

Para superar estas dificultades algunos autores han propuesto el término "metagobernanza" para referirse a formas soft de asegurar la regulación de espacios reticulares (Kooiman, 1993; Jessop, 2002). La meta-gobernanza establece los límites del proceso decisional, aunque sea aceptando el espacio del marco de referencia al cual nos referíamos anteriormente. En palabras de Scharpf (1994: 40), la gobernanza [deliberativa] siempre se produce "in the shadow of hierarchy". 
La política y los políticos, en conclusión, siguen desempeñando un papel imprescindible en el desarrollo de las políticas públicas en un contexto de elevada complejidad. No sólo por su capacidad de desbloquear situaciones donde el conflicto es el estado natural sino, también, porque representan el nexo de unión con la legitimidad democrática para tomar decisiones dotadas de autoridad. Sorensen \& Torfing (2005) se refieren a este nexo como el "anclaje democrático". Nosotros hemos usado este anclaje para mostrar la necesaria relación entre deliberación y autoridad.

\section{Bibliografía}

Axeldrod, R. y Cohen, M. H. (1999). Harnessing complexity. Organizational implications of scientific frontier. Free Press, New York.

Bauman, Z. (2000). Liquid Modernity. Polity, Londres.

Barber,B. (2004). Democracia Fuerte. Almazara, Granada.

Beck, U. (1998). La Sociedad del Riesgo Global. Madrid: Siglo XXI.

Benhabib, S. (1996). Democracy and Difference: Contesting the Boundaries of the Political. Princeton University Press, Princeton.

Bovens, M. y Hard, P.Y. (1998). Understanding Policy Fiascoes. Transaction Press, New Brumswick.

Brugué, Q. (2009). "Una Administración que Habla es una Administración que Piensa", en AA.VV. Participación Ciudadana para una Administración Deliberativa. Gobierno de Aragón, Zaragoza.

Brugué, Q. (2013). Política de Agua con Diálogo Social. La Aplicación de la DMA en Catalunya. Informe final del proyecto CSO2009-09880, pendiente de publicación.

Castells, M. (1996). La Era de la Información. Economía, Sociedad y Cultura. Alianza, Madrid.

Cilliers,P. (2005). "Complexity, Deconstruction and Relativism", en Theory, Culture \& Society, vol. 22, no 5 , pp. 255-267.

Dryzek, J. (2000). Deliberative Democracy and Beyond. Oxford University Press.

Espluga,J, L. et al. (2011). "Agua, Participación e Inercia Institucional”, en Revista Española de Investigaciones Sociológicas, $\mathrm{n}^{\circ} 134$, pp. 3-26.

Fischer, F. y Forester, J. (eds). (1993). The Argumentative Turn in Policy Analysis and Planning. Duke University Press, Durham.

Fischer, F. (2003). Reframing Public Policy. Oxford University Press, Oxford.

Generalitat de Catalunya. (2006). Del Aqui No al Así Sí. Generalitat de Catalunya, Barcelona.

Gutmann, A. y Thompson,D. (2004). Why Deliberative Democracy? Princeton University Press, Princeton.

Habermas, J. (1999). La inclusión del Otro. Estudios de Teoría Política. Paidós, Barcelona.

Hajer, M. y Wagennar, H. (2003). Deliverative Policy Analysis: Understanding Governance in the Network Society. Cambridge University Press, Cambridge.

Hay, C. (2007). Why We Hate Politics. Polity, London.

Healey, P. (1997). Collaborative Planning: Shaping Places in Fragmented Societies. Macmillan, London. 
Holland, J. H. (1998). Emergence: from Cahos to Order. Oxford University Press, Oxford.

Innerarity, D. (2011). La Democracia del Conocimiento. Paidós, Barcelona.

Kettl, D. (2002). The Transformation of Governance. The Johns Hopkins University Press, Baltimore.

Jessop, B. (2002). The Future of the Capitalist State. Polity Press, Cambridge.

Kooiman, J. (1993). Modern Governance: New Government-Society Interactions. Sage, London.

Mansbridge, J. (1983). Beyond Adversary Democracy. Chicago University Press.

Martí, J. L. (2006). La República Deliberativa. Una Teoría de la Democracia. Marcial Pons, Barcelona.

Nel·lo, O. (ed). (2003). Aquí, No! Els Conflictes Territorials a Catalunya. Empúries, Barcelona.

O’Leary, R. y Bingham, L. B. (2009) The Collaborative Public Manager. Georgetown University Press, Washington, D. C.

O’Leary, R., Van Slyke, D. y Kim, S. (ed.). (2010). The Future of Public Administration Around the World. Georgetown University Press, Washington, D. C.

Ovejero, F. (2013). ¿Idiotas o Ciudadanos? Montesinos, Barcelona.

Pierre, J. (ed.). (2000). Debating Governance: Authority, Steering and Democracy. Oxford University Press.

Pollit, C. (2003). The Essential Public Manager. Open University Press, Buckingham.

Rhodes, R.A.W. (1997). Understanding Governance. Policy Networks, Governance and Accountability. Open University Press, Buckinham.

Scharpf, F. (1994). "Games Real Actors Could Play: Positive and Negative Coordination in Embedded Negotiations", en Journal of Theoretical Politics, $\mathrm{n}^{\circ}$ 6, pp. 27-53.

Sorensen, E. y Torfing, J. (2005). "The Democratic Anchorage of Governance Networks", en Scandinavian Politica Studies, vol. 28, n 39, pp. 195-218.

Vallès, J. M. (2011). "Política: Una Reivindicació a Contra Corrent", en Llección inagural del curso académico 2011-2012, Universitat Autònoma de Barcelona.

Wacquant, L. (2006) Castigar els Pobres. Edicions de 1984, Barcelona.

Wagennar, H. (2007). "Governance, Complexity and Democratic Participation", en The American Review of Public Administration, n 37, pp. 17-50. 